\title{
An Automatic Collision Detection and Avoidance Module for Inland Navigation
}

\author{
Alexander Lutz* Ernst-Dieter Gilles ** \\ * Institut für Systemdynamik, Universität Stuttgart, Pfaffenwaldring 9, \\ 70569 Stuttgart, Germany (e-mail: lutz@isys.uni-stuttgart.de) \\ ** Max-Planck-Institut Magdeburg, Sandtorstr. 1, 39106 Magdeburg, \\ Germany(e-mail: gilles@mpi-magdeburg.mpg.de)
}

\begin{abstract}
Based on the positional data of other inland vessels which is available through the fusion of radar object tracking and AIS (Automatic Identification System) a model-based prediction of their kinematic states is presented. Information about the river shape is fed into the prediction model according to the type of vessel and its navigational situation. Together with the future states of our own vessel, which are known because it is automatically steared along a guiding line, ranges of encounter are calculated. Furthermore, a path planning algorithm based on nonlinear dynamic optimization is presented that is capable of calculating collision-free paths in real-time that account for the vessel's constrained dynamics. Practical results of the discussed automatic collision detection and avoidance module as part of an integrated navigation system for inland vessels are shown concluding the contribution.
\end{abstract}

Keywords: Model-based Prediction, Path Planning, Nonlinear Optimization, Path Following

\section{INTRODUCTION}

At the Institute for System Dynamics (ISYS), the former Institute for System Dynamics and Control (ISR), at the University of Stuttgart an integrated navigation system for inland vessels has been developed over the last three decades. It is capable of displaying electronic navigational charts (ENC), standardized according to InlandECDIS (Electronic Chart Display and Information System) (see Zentralkommission für die Rheinschifffahrt (2006)) combined with overlayed radar. A map-matching algorithm rotates the ENC such that it matches the overlayed radar image. For that reason, characteristic features such as shore lines are extracted from both, the ENC and the radar image, and brought into accordance using a least-squares method as shown by Sandler (2004). The radar image is also used to estimate the dynamic states of other objects on the river using radar object tracking. By extracting specific points such as the center of area from areas resembling radar echoes on the river, conclusions about the movement of the tracked objects can be drawn if several radar images are compared. Plocher and Gilles (1992) and Gern (2000) explain the radar object tracking algorithms in detail. Section 2.1 introduces the kinematic model that is used in the Kalman filter which is responsible for radar object tracking and data fusion with GNSS (Global Navigation Satellite System) data transmitted through AIS. An asynchronous Kalman filter algorithm is employed to combine data from various sensors such as the turning rate of the vessel from a gyroscope, the velocity, course and heading from a GPS (Global Positioning System) compass and the rudder angle from a rudder angle indicator in order to estimate the dynamic state of the vessel.

A peculiar feature of this integrated navigation system is the automatic track-keeping capability. The vessel's rudder angle is controlled in order to stear the vessel along a given guiding line. A feedforward rudder angle is computed from the twice continuously differentiable guiding line by inverting the dynamic model of the ship. A Riccati-based feedback rudder angle compensates for disturbances and model uncertainties. This control system is able to keep the cross-track control error of arbitrary inland vessels below $3 m$ std.

Section 2 describes the collision detection functionality by first introducing the dynamic model that is used to fuse the radar object information with AIS data. A simplified version of this kinematic model is used in section 2.2 in order to predict the other vessels' states in the future by utilizing information about the shape of the river. Time-independent ranges of encounter are calculated in section 2.3 based on these predictions together with the information about our vessel's future position.

Section 3 presents the collision avoidance algorithm. It shows how to utilize nonlinear programming to compute collision-free paths. Section 3.1 introduces a simplified ship model, the so-called setpoint filter model, to account for the vessel's dynamics. The black-box optimization package NOMAD (see Abramson et al. (2009)) is used to calculate the solutions that are shown in section 3.1.4.

Section 4 concludes this contribution by comparing the different avoidance approaches and discussing issues arising in practice.

\section{COLLISION DETECTION}

In order to compute collision-free paths the navigational environment has to be known. Besides stationary objects like bridge pillars, radar buouys and fairway boundaries information about moving obstacles like other vessels is required. While most stationary data can be retrieved from 
the electronic charts information about moving objects has to be generated from measurements using the radar image and AIS data if available. Means to predict the moving objects' dynamic states are necessary in order to compute their future positions at times when our vessel is next to them. These ranges of encounter must be avoided by the path planning algorithm in order to compute collision-free paths.

\subsection{Radar Object Tracking and AIS Data Fusion}

Every inland vessel is required to have a radar antenna with the corresponding radar equipment. The radar serves as the main sensor to collect data about the navigational environment including the shore line and especially other vessels. The integrated navigation system is able to track other vessels by analyzing several consecutive radar images. In order to do that, geometric features of radar echoes that lie within the river are extracted and fed into a Kalman filter corresponding to the tracked object. The position of the center of area of the radar echo can be used as such a geometric feature. A way to also gain heading information besides pure positional information is to map every radar echo onto a suitable ellipse. The two points from the intersection of the major axis with the ellipse serve as the positions of bow $\boldsymbol{B}=\left(B_{x}, B_{y}\right)^{T}$ and stern $\boldsymbol{S}=\left(S_{x}, S_{y}\right)^{T}$. These positions are fed into a Kalman filter that uses the following purely kinematic model from Gern (2000):

$$
\begin{aligned}
\dot{B}_{x} & =v_{x} \\
\dot{B}_{y} & =v_{y} \\
\dot{S}_{x} & =v_{x} \\
\dot{S}_{y} & =v_{y} \\
\dot{v}_{x} & =-r v_{y} \\
\dot{v}_{y} & =r v_{x} \\
\dot{r} & =0 .
\end{aligned}
$$

$B_{x}, B_{y}, S_{x}, S_{y}$ denote positions in an earth-fixed coordinate system with the $\mathrm{x}$-axis facing north and the $\mathrm{y}$-axis facing east. $v_{x}$ denotes the object's velocity in northern direction whereas $v_{y}$ is the velocity in eastern direction. $r$ describes the turning rate about the $\mathrm{z}$-axis which is facing down. The bow's velocity is assumed to be equal to the stern's velocity. This assumption contradicts the fact that the turning rate $r$ is not zero. Nevertheless, this model still leads to reasonable estimates. Whenever the position of the bow $\boldsymbol{B}$ and the stern $\boldsymbol{S}$ are measured the Kalman filter states are updated. Since both measurements are fed into the Kalman filter synchronously the estimated velocity will end up being the mean of the real velocities at the bow and stern. Thus, the estimated velocity $\left(v_{x}, v_{y}\right)^{T}$ describes the velocity at the center of the vessel since the vessel is a rigid body. That way, the estimation gains robustness against symmetric variations in the length of the major axis of the ellipse.

If AIS data is available a third point $\boldsymbol{A}=\left(A_{x}, A_{y}\right)^{T}$ for the AIS positional measurements can be added. By including this third point, the velocity vector $\left(v_{x}, v_{y}\right)^{T}$ and ultimately the turning rate $r$ get updated when an AIS measurement becomes available. Therefore, the information from the radar antenna and the AIS receiver is fused by this Kalman filter to get a combined estimate.

Typical values for the standard deviations of this radar object tracking method without AIS fusion are given in table 1.

Table 1. Std for the radar object tracker

\begin{tabular}{ccc} 
Position $(\mathrm{m})$ & Velocity $(\mathrm{m} / \mathrm{s})$ & Turning Rate $\left({ }^{\circ} / \mathrm{min}\right)$ \\
\hline 6.6 & 0.23 & 1.7 \\
\hline
\end{tabular}

\subsection{State Prediction}

In order to predict the future dynamic states of other vessels a single point kinematic model is used. It is the same as the two point model from the previous section with the difference of only having one point, namely the center of area of the vessel's projection onto the xy-plane:

$$
\begin{aligned}
\dot{x} & =v_{x} \\
\dot{y} & =v_{y} \\
\dot{v_{x}} & =-r v_{y} \\
\dot{v_{y}} & =r v_{x} \\
\dot{r} & =0
\end{aligned}
$$

If this model is initialized with values from the radar object tracker it can be integrated in order to receive future values of the vessel's position under the assumption that the turning rate $r$ remains constant. However, this prediction method exhibits two major drawbacks:

(1) Initial uncertainties are increased through integration.

(2) The shape of the river is not taken into account.

(1) Using a linearized version of model (8)-(12) it can be shown that the initial uncertainties given in table 1 lead to a standard deviation of the position of $35 \mathrm{~m}$ after $100 s$ already. This means that the position circle for the estimate with a confidence of $95.5 \%(2 \sigma)$ already has a diameter of $140 \mathrm{~m}$ which is more than the width of most fairways in Europe.

(2) If a vessel's future position is estimated with the described method while it is on a straight river stretch in front of a bend the estimate will result in a straight line and will therefore leave the bending river. This is due to the fact that the information about the river bend is not visible in the estimated turning rate yet.

Problems (1) and (2) lead to the conclusion that additional information has to be included to back up the prediction method. A skipper is still able to predict other vessels' behaviors although his estimates of their dynamic states are certainly not more accurate than the Kalman filter estimates. This is due to the fact that a skipper uses his knowledge about typical navigational routes and traffic rules on top of the initial position and velocity. That is why information from guiding lines which are available in the integrated navigation system is used to increase the prediction accuracy. Guiding lines serve as the reference tracks for the automatic track-keeping module and represent therefore typical navigational routes. Several guiding lines exist for different water levels and for the direction of navigation (upstream or downstream). These lines also 
implicitly contain information about the shape of the river since they lie within the river's fairway.

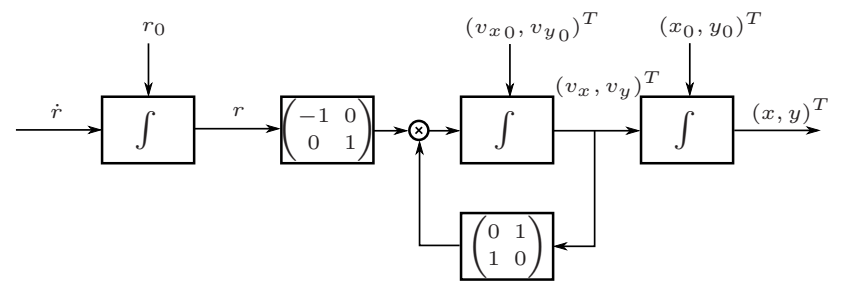

Fig. 1. Block diagram of the prediction model.

The information about the river shape is fed into the prediction model at different places depending on the initial state of the vessel. Figure 1 shows the block diagram of the prediction model (8)-(12). The later the information about the guiding line is injected into the prediction model the more the initial uncertainties are reduced. However, the assumption that the vessel travels along the guiding line is dominating more and more over the integration of the inital estimates which is not always desirable. The following possibilities to inject guiding line information are used:

(1) $\dot{r}$ from the guiding line: The second time derivative of the course angle $\ddot{\alpha}$ that is required to stay on the guiding line if the initial values for $r$ and $\boldsymbol{v}$ are in accordance with the guiding line is given as

$$
\ddot{\alpha}=v_{a b s}^{2} \kappa_{G L}^{\prime}
$$

with the absolute velocity $v_{a b s}$ and the guiding line's curvature derivative $\kappa_{G L}^{\prime}$. Under the assumption that the second time derivative of the drift angle $\ddot{\beta}$ is zero, the time derivative of the turning rate for traveling along the guiding line is given as

$$
\dot{r}=v_{a b s}^{2} \kappa_{G L}^{\prime} .
$$

Note: $\dot{\psi}=r$ and $\psi=\alpha+\beta$, with $\psi$ being the heading angle, $\alpha$ being the course angle and $\beta$ being the drift angle.

(2) $r$ from the guiding line: To further reduce the influence of initial uncertainties it is assumed in this case that the turning rate of the predicted vessel follows the one that is required to navigate along the guiding line. For kinematic reasons

$$
\dot{\alpha}=v_{a b s} \kappa_{G L}
$$

has to hold which means that

$$
r=v_{a b s} \kappa_{G L}
$$

has to be valid under the assumption of a negligible drift derivative $\dot{\beta}$.

(3) $\boldsymbol{v}_{0}$ projected onto the guiding line: Under the assumption that the vessel travels along the guiding line its initial velocity vector $\boldsymbol{v}_{0}=\left(v_{x 0}, v_{y 0}\right)^{T}$ is projected onto the guiding line such that the resulting velocity vector is tangential to the guiding line. Furthermore, the turning rate $r$ from the guiding line is fed into the prediction model. This prediction results in a line parallel to the guiding line.

According to the type of vessel and its initial dynamic state, one of the prediction methods $(1)-(3)$ is chosen.
For example, it is clear that prediction method (3) is not valid for a ferry traveling across the river with a large angle between its course and the guiding line. Figure 2 shows the prediction errors for the different prediction methods for a ferry crossing the river. The values are calculated from a real world scenario. Method 0 denotes pure integration whereas method 4 denotes a special variant of method 3 with an initial angle to the guiding line that is reduced with first order dynamics.

While prediction method (3) performs poorly when predicting ferries it works very well for normal vessels traveling on the river. Figure 3 depicts such a case. Generally speaking, a set of conditions determine the choice of the prediction method. The conditions consisit of the angle between the course and the guiding line, the existence of ferry lines and the existence of lane switching areas where the standard navigation side for up- and downstream traffic is switched.

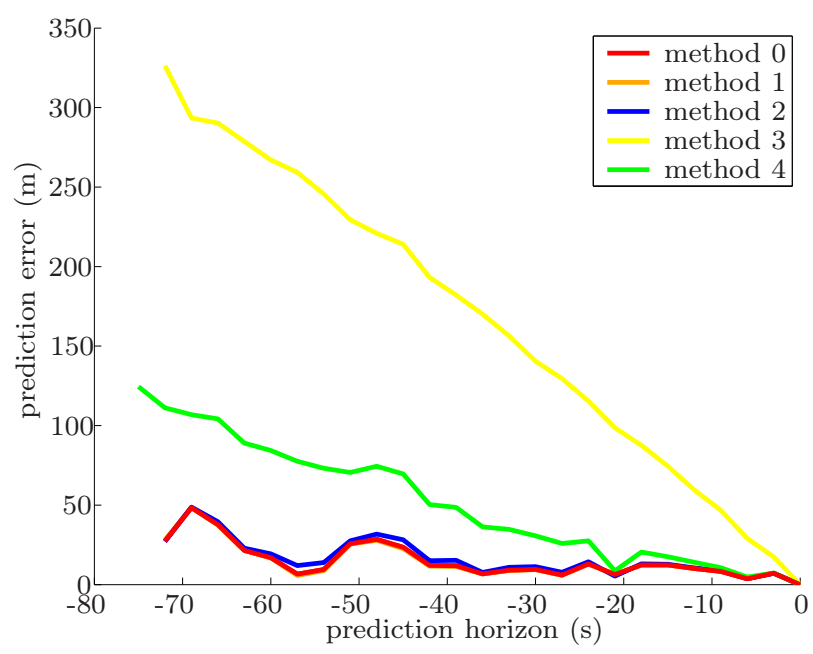

Fig. 2. Prediction errors for the different prediction methods for a ferry crossing the river.

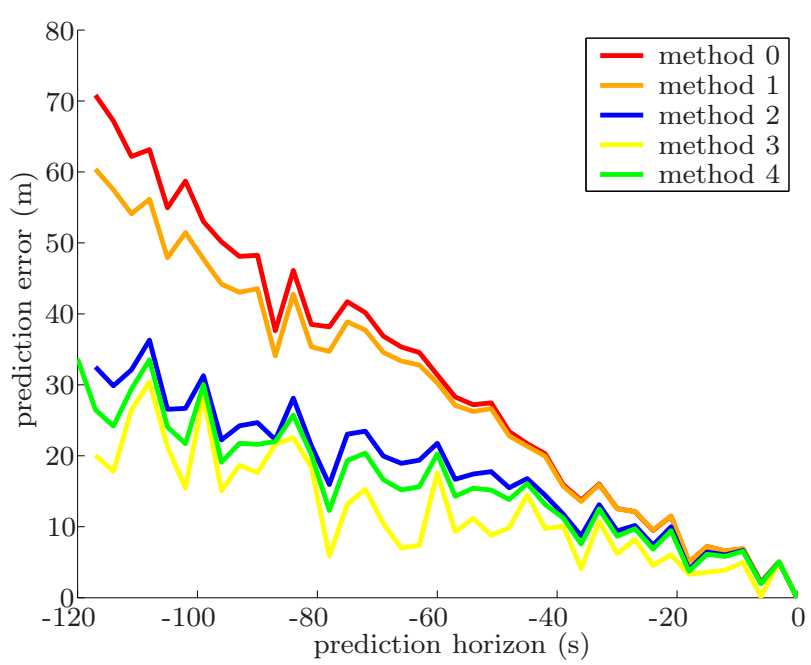

Fig. 3. Prediction errors for the different prediction methods for a normal vessel. 


\subsection{Ranges of Encounter}

A range of encounter is defined as the area on the river occupied by another object while our vessel is traveling next to it along the river. Ranges of encounter are spatial objects and do not exhibit a time component. Therefore, they are locally fixed. Planned paths can be considered collision-free if the spatial dimensions of our vessel do not intersect any range of encounter. In order to treat our vessel as one point only, namely the center of area, the ranges of encounter are enlarged to account for our vessel's dimensions.

Beginning of encounter

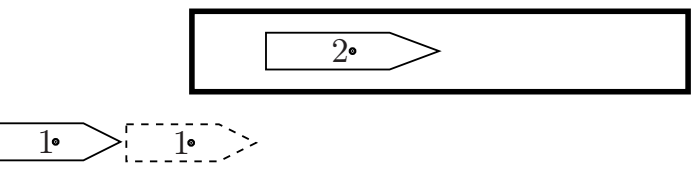

End of encounter

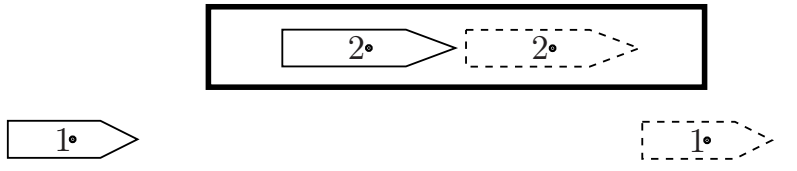

Fig. 4. Range of encounter for passing drawn for the center of area of vessel 1 .

Figure 4 shows the generation of such a range of encounter using a passing procedure of vessel 1 as example. Since the relative velocity between the two vessels determines the amount of time during which both vessels are next to each other the range of encounter in the example is longer than the length of vessel 2. Therefore, in case of a stationary object the range of encounter equals the vessel's dimensions plus the enlargement for our own vessel whereas the range of encounter is smaller than the vessel size plus enlargement when the two vessels meet with velocities greater than zero.

For all types of encounter the beginning and the end of the encounter are characterized by either the bow or stern of both vessels being at the same point along the river. Therefore, there exist four possibilities: bow-bow, bowstern, stern-bow, stern-stern. By introducing a coordinate system whose $\mathrm{x}$-axis points along the river in the direction of our vessel's velocity, the z-axis down and the y-axis such that a right hand system is formed the bow and stern of our vessel at $t=t_{0}$ is given as

$$
\begin{aligned}
& x_{e 1}\left(t_{0}\right)=x_{e}\left(t_{0}\right)+\frac{L_{e}}{2}, \\
& x_{e 2}\left(t_{0}\right)=x_{e}\left(t_{0}\right)-\frac{L_{e}}{2},
\end{aligned}
$$

with $L_{e}$ being our vessel's length. Similarly, for the other vessel:

$$
\begin{aligned}
& x_{o 1}\left(t_{0}\right)=x_{o}\left(t_{0}\right)+\frac{L_{o}}{2}, \\
& x_{o 2}\left(t_{0}\right)=x_{o}\left(t_{0}\right)-\frac{L_{o}}{2},
\end{aligned}
$$

with its length $L_{o}$. Denoting the velocity along the x-axis of our vessel by $v_{x e}$ and the other vessel's velocity by $v_{x o}$, the four possible extrema are calculated by

$$
\begin{aligned}
t_{E 1} & =\frac{x_{o 1}\left(t_{0}\right)-x_{e 1}\left(t_{0}\right)}{v_{x e}-v_{x o}}, \\
t_{E 2} & =\frac{x_{o 1}\left(t_{0}\right)-x_{e 2}\left(t_{0}\right)}{v_{x e}-v_{x o}}, \\
t_{E 3} & =\frac{x_{o 2}\left(t_{0}\right)-x_{e 1}\left(t_{0}\right)}{v_{x e}-v_{x o}}, \\
t_{E 4} & =\frac{x_{o 2}\left(t_{0}\right)-x_{e 2}\left(t_{0}\right)}{v_{x e}-v_{x o}} .
\end{aligned}
$$

Which two of the four extrema are active is determined by the following equations:

$$
\begin{aligned}
& T_{E 1}=\min \left(t_{E 1}, t_{E 2}, t_{E 3}, t_{E 4}\right), \\
& T_{E 2}=\max \left(t_{E 1}, t_{E 2}, t_{E 3}, t_{E 4}\right),
\end{aligned}
$$

with $T_{E 1}$ being the beginning of the encounter and $T_{E 2}$ being the end. The spatial dimensions of the range of encounter can easily be calculated as $x_{e}\left(T_{E 1}\right)$ and $x_{e}\left(T_{E 2}\right)$ in $\mathrm{x}$-direction and as $y_{o}\left(T_{E 1}\right)$ and $y_{o}\left(T_{E 2}\right)$ in y-direction extended by the widths of the other vessel and our vessel. Note: These derivations are only valid for the standard case of a vessel traveling along the river. They are not valid for vessels going across the river such as ferries. Such vessels must be treated separately. The new coordinate system's $\mathrm{x}$-axis follows the river and is therefore not straight. Nevertheless, the coordinate system can be treated as a cartesian system locally since the typical curvature of rivers is small enough.

The ranges of encounter are also enlarged in order to account for drift angles of the other vessels and our own vessel. A worst-case assumption on the possible drift angles is adopted but omitted here.

\section{COLLISION AVOIDANCE}

A path planning algorithm for inland navigation must satisfy certain criteria if the resulting path will be used as reference track for automatic track-keeping.

(1) Navigability: The vessel which the path is computed for must be able to follow the path. This means that the required rudder angle $\delta$ cannot exceed a maximum value. The constrained dynamics of the rudder hydraulics can be accounted for by also requiring bounds on the time derivative of the rudder angle $\dot{\delta}$.

(2) State constraints: Bounds on the turning rate $r$ and the angle between the course $\alpha$ and the guiding line ensure that the avoidance module computes reasonable paths. They can be freely chosen by the captain and pose a trade-off between smooth avoidance paths and more extreme maneuvers.

(3) Collision-free paths: The generated paths must be collision-free. All ranges of encounter have to be avoided as well as the boundaries of the fairway.

(4) Maneuver detectability: Other vessels need to be able to realize what type of avoidance maneuver our vessel is carrying out in order to take measures accordingly. This means that the calculated paths must result in 
short clear maneuvers as opposed to long transient behavior.

\subsection{Path Planning using Nonlinear Optimization and the Setpoint Filter Model}

Path planning based on nonlinear optimization using the so-called setpoint filter model is able to account for all criteria given above. Navigability is inherintly included in the special model. The optimization algorithm is capable of dealing with state constraints as well as constraints that guarantee collision-free paths. The resulting maneuvers are also detectable since this avoidance algorithm only permits a limited number of clear lane changes.

Mathematical Model The setpoint filter model is based on a reduced ship model and was first introduced by Wahl (2001). Its state vector consists of the turning rate $r$, its time derivative $\dot{r}$ the heading angle $\psi$ between the vessel and the guiding line and the distance to the guiding line $y$. The model equations read as follows:

$$
\begin{aligned}
\frac{d}{d t}\left(\begin{array}{c}
\dot{r} \\
r \\
\psi \\
y
\end{array}\right) & =\left(\begin{array}{c}
-C_{4} \dot{r}-C_{3} r+C_{3} r_{o f f} \\
\dot{r} \\
r \\
L_{D} r+u \psi
\end{array}\right), \\
d_{f} & =y,
\end{aligned}
$$

with

$$
\begin{aligned}
& r_{o f f}=\operatorname{sat}\left(\frac{C_{2}}{C_{3}}\left(\psi_{o f f}-\psi\right), r_{\max }\right), \\
& \psi_{o f f}=\operatorname{sat}\left(\frac{C_{1}}{C_{2}}\left(d_{o f f}-y\right), \psi_{\max }\right) .
\end{aligned}
$$

The input to this model is $d_{\text {off }}$ and represents the desired lateral offset to the guiding line. The model structure is similar to a fourth-order lag system except for the fact that the saturation functions (29) and (30) limit the turning rate $r$ to $r_{\max }$ and the heading to the guiding line $\psi$ to $\psi_{\max }$. The output $d_{f}$ is a filtered lateral offset that assymptotically tends to $d_{o f f}$. Equation (27) can be viewed as a ship model with linearized lateral dynamics and a continuous turning acceleration $\dot{r}$. The lateral dynamics consist of the term $u \psi$ which represents the lateral component of the constant forward speed $u$ and the drift term $L_{D} r$ with $L_{D}$ as the distance between the pivot point and the center of gravity. A suitable choice of the free parameters $C_{1}, \ldots, C_{4}$ is given by Wahl (2001).

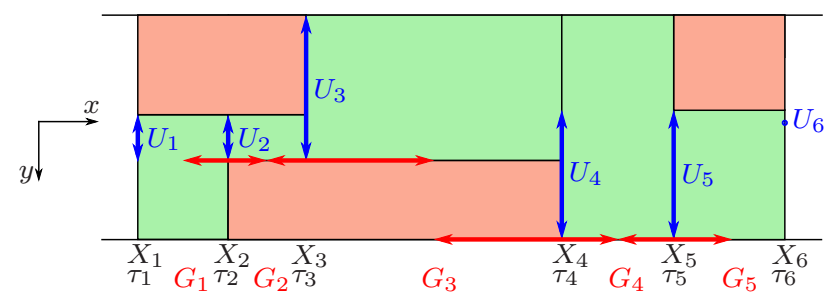

Fig. 5. Avoidance scenario with constraints of the variable lateral offset inputs $U_{i}$ with variable switching times $\tau_{i}$.

Problem Formulation Figure 5 depicts a typical avoidance scenario. The ranges of encounter are drawn as red boxes whereas the navigable part of the river is shown in green. All coordinates refer to the guiding line coordinate system that was introduced above with the $\mathrm{x}$-axis pointing in guiding line direction. Since our vessel's velocity in $\mathrm{x}$-direction is considered constant all coordinates in $\mathrm{x}$ direction can be transformed into time coordinates and vice versa. At every $\mathrm{x}$-position $X_{1}-X_{6}$ a new input $U_{1}-U_{6}$ to the setpoint filter model can be chosen by the optimization algorithm. By limiting the number of offsets the avoidance maneuvers become detectable. One offset can be chosen at the before each range of encounter and after each range. Each offset's $U_{i}$ upper and lower bound is determined by the available lateral space at the next offset. The last offset $U_{6}$ is chosen to be zero which means that the avoidance path ends on the guiding line again. With these definitions the optimization problem can be formulated as follows:

The goal is to find the piecewise constant input function $u^{*}(t, \boldsymbol{\tau})$ with

$$
u(t, \tau)=\left\{\begin{array}{ll}
U_{i} & \text { for } \tau_{i}<t<\tau_{i+1} \\
0 & \text { for } t>\tau_{n_{f}+1}
\end{array} \quad \forall i=1 \ldots n_{f}\right.
$$

and

$$
\begin{gathered}
\tau_{1}=\frac{1}{v_{x}}\left(X_{1}-x_{0}\right), \\
\tau_{n_{f}+1}=\frac{1}{v_{x}}\left(X_{n_{f}+1}-x_{0}\right), \\
\tau_{i} \in\left(G_{i-1}, G_{i}\right) \quad \forall i=2, \ldots, n_{f}, \\
G_{i}=\frac{1}{v_{x}}\left(0,5\left(X_{i+1}-X_{i}\right)-x_{0}\right),
\end{gathered}
$$

that minimizes the cost function

$$
J=\Phi(\boldsymbol{x}(t), u(t, \boldsymbol{\tau}))
$$

such that equation (27), the initial conditions

$$
\dot{r}\left(t_{0}\right)=\dot{r}_{0}, r\left(t_{0}\right)=r_{0}, \psi\left(t_{0}\right)=\psi_{0}, y\left(t_{0}\right)=y_{0},
$$

the input constraints

$$
\begin{gathered}
l b_{i} \leq u(t, \boldsymbol{\tau}) \leq u b_{i} \\
\frac{1}{v_{x}}\left(X_{i}-x_{0}\right)<t<\frac{1}{v_{x}}\left(X_{i+1}-x_{0}\right), \\
\forall i=1 \ldots n_{f}
\end{gathered}
$$

with

$$
\begin{gathered}
l b_{i}=\max \left(y_{\min , i}, y_{\min , i+1}\right), \quad \forall i=1 \ldots n_{f}-1 \\
u b_{i}=\min \left(y_{\max , i}, y_{\max , i+1}\right), \quad \forall i=1 \ldots n_{f}-1 \\
l b_{n_{f}}=y_{\min , n_{f}} \\
u b_{n_{f}}=y_{\max , n_{f}}
\end{gathered}
$$

and the output constraints 


$$
\begin{gathered}
y_{\min , i} \leq d_{f}(t) \leq y_{\max , i} \\
\frac{1}{v_{x}}\left(X_{i}-x_{0}\right)<t \leq \frac{1}{v_{x}}\left(X_{i+1}-x_{0}\right) \\
\forall i=1 \ldots n_{f}
\end{gathered}
$$

are satisfied. In this problem formulation each of the $n_{f}$ green zones is described by the four-tuple

$$
\left\{x_{\min }, x_{\max }, y_{\min }, y_{\max }\right\}_{i} \quad \forall i=1 \ldots n_{f} .
$$

The choice of the cost function $J$ (36) strongly influences the shape of the avoidance path. Two possible choices are:

(1) Minimization of the distance to the guiding line:

$$
J=W_{y} \int_{t_{0}}^{t_{f}} d_{f}^{2}(t) d t+W_{u} \sum_{i=1}^{n_{f}-1}\left(U_{i+1}-U_{i}\right)^{2} .
$$

(2) Maximization of the distance to obstacles:

$$
\begin{aligned}
& J=W_{y} \int_{t_{0}}^{t_{f}}\left(d_{f}(t)-0,5(l b(t)+u b(t))^{2} d t+\right. \\
& W_{u} \sum_{i=1}^{n_{f}-1}\left(U_{i+1}-U_{i}\right)^{2}, \quad \text { with } \\
& l b(t)=l b_{i}, \quad u b(t)=u b_{i}, \quad \text { and } \\
& t \in\left[\frac{1}{v_{x}}\left(X_{i}-x_{0}\right), \frac{1}{v_{x}}\left(X_{i+1}-x_{0}\right)\right) \forall i=1 \ldots n_{f} .
\end{aligned}
$$

Both choices for the cost function $J$ include a term punishing the difference between consecutive inputs besides the term describing the primary goal. This term allows for a trade-off between the primary goal and a straighter path by adjusting the corresponding weights $W_{y}$ and $W_{U}$.

Problem Solution The problem (31)-(47) is discretized using a forward Euler integration method for the differential equation with a sufficiently small sample time to guarantee numerical stability. The nonlinear optimization package NOMAD belongs to the group of black-box optimization tools. This means that only the cost function value depending on the optimization variables is needed to find the solution to the optimization problem. Derivatives for the cost function and the constraints are not necessary. It is capable of solving non-smooth nonlinear optimization problems with a small number of variables.

Since the inputs to our optimization problem only change $n_{f}$ times it is sufficient to only use the vector $\left(U_{1}, \ldots, U_{n_{f}}\right)^{T}$ as optimization variables in addition to $n_{f}-1$ variables $l_{i}$ that denote the variable switching times of $U_{2}, \ldots, U_{n_{f}}$.

Results Figure 6 shows a high traffic scenario with six stationary objects together with the resulting collision-free paths that are computed using the nonlinear optimization problem with the cost function variants (1) and (2). Corresponding values for the number of optimization variables and the computation times are given in table 2 .

\section{CONCLUSION}

In practice the presented collision detection and avoidance method robustly delivers solutions for any relevant problem size.

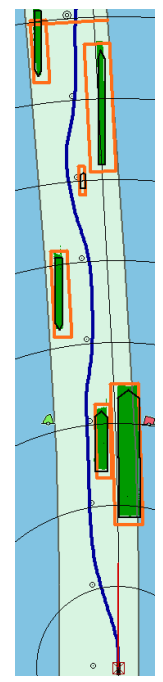

(a) $J=(1)$

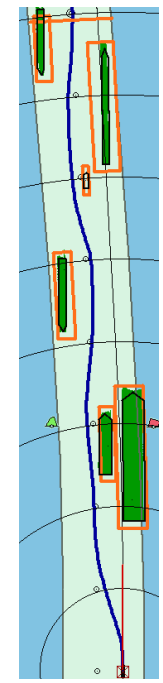

(b) $J=(2)$
Fig. 6. Avoidance paths for a high traffic scenario for both version of the cost function $J$.

Table 2. Number of optimization variables and computation time for scenario in figure 6 .

\begin{tabular}{cccccc} 
Fig & $J$ & $W_{y}$ & $W_{u}$ & Variables & Time $(\mathrm{ms})$ \\
\hline $6(\mathrm{a})$ & $(1)$ & 100 & 100 & 17 & 1361 \\
$6(\mathrm{~b})$ & $(2)$ & 100 & 100 & 17 & 1400 \\
\hline
\end{tabular}

The fairly large uncertainties described in section 2 need to be compensated for by repeatedly solving the avoidance problem. The results show that the computation time that is needed to solve one problem permits the repeated solution at update rates greater than $0.5 \mathrm{~Hz}$ which is sufficient considering the vessels' velocities.

Ferries and other vessels crossing the river can not be accounted for with the presented algorithm. They pose a special problem also because they are recognized fairly late by the radar object tracker. This is due to the fact that the radar object tracker needs several radar images as measurements for the recognition.

\section{REFERENCES}

Abramson, M.A., Audet, C., Couture, G., Dennis, J.E., and Digabel, S.L. (2009). The nomad project. Software available at http://www.gerad.ca/nomad.

Gern, T. (2000). Automatische Flußkartengenerierung. Logos Verlag Berlin.

Plocher, T. and Gilles, E.D. (1992). Rekursive Objektverfolgung in Bildsequenzen. Automatisierungstechnik, 40, 14-20,59-63.

Sandler, M. (2004). Integrierte Messdatenverarbeitung in einem Navigationssystem für Binnenschiffe. Logos Verlag Berlin.

Wahl, A. (2001). Einsatz optimaler Regelverfahren zur automatischen Bahnführung. VDI Verlag GmbH.

Zentralkommission für die Rheinschifffahrt (2006). Inland ECDIS Standard Edition 2.0. http://www.ccr-zkr.org. 